\title{
Utilization of geoinformation tools for the development of forest fire hazard mapping system: example of Pekan fire, Malaysia
}

\begin{abstract}
A study in modeling fire hazard assessment will be essential in establishing an effective forest fire management system especially in controlling and preventing peat fire. In this paper, we have used geographic information system (GIS), in combination with other geoinformation technologies such as remote sensing and computer modeling, for all aspects of wild land fire management. Identifying areas that have a high probability of burning is an important component of fire management planning. The development of spatially explicit GIS models has greatly facilitated this process by allowing managers to map and analyze variables contributing to fire occurrence across large, unique geographic units. Using the model and its associated software engine, the fire hazard map was produced. Extensive avenue programming scripts were written to provide additional capabilities in the development of these interfaces to meet the full complement of operational software considering various users requirements. The system developed not only possesses user friendly step by step operations to deliver the fire vulnerability mapping but also allows authorized users to edit, add or modify parameters whenever necessary. Results from the model can support fire hazard mapping in the forest and enhance alert system function by simulating and visualizing forest fire and helps for contingency planning.
\end{abstract}

Keyword: Forest fire hazard; GIS; Malaysia; Pekan 